\title{
AUTONOMOUS ORBIT CONTROL PROCEDURE, USING A SIMPLIFIED GPS NAVIGATOR AND A NEW LONGITUDE PHASE DRIFT PREDICTION METHOD, APPLIED TO THE CBERS SATELLITE
}

\author{
Roberto Luiz Galski (1), Valcir Orlando (2) \\ (1) (2) CRC/INPE, Av. Astronautas 1758, São José Campos, SP, Brazil, (55)12-3208-6386, \\ galski@ccs.inpe.br,(55)12-3208-6386,valcir@ccs.inpe.br
}

\begin{abstract}
This paper presents a performance analysis of an autonomous orbit control procedure using a simplified GPS navigator [1], where the ground track drift of the satellite is estimated onboard with help of a recently developed approach [2] that directly calculates the acceleration of the orbit ground track as a function of the solar and geomagnetic activity. The simplified navigation procedure improves the coarse geometric navigation solution provided by GPS receivers. This is done by using the GPS solutions as inputs (observations) for a real time Kalman filtering process. The orbital state vector is extended and includes the systematic error imposed to the GPS geometric solution by the changes in the set of satellites which are visible to the receiver. The simplified navigator has reduced computational cost, allowing it to be carried and executed on-board of spacecrafts. The improved outputs of this process are used in the computational implementation of an autonomous control system for the ground track drift of the spacecraft orbit. The behavior of the system is evaluated by means of orbit simulations using a CBERS-like phased remote sensing satellite. The aim of the paper is to verify if the coupled system is able to correctly calculate and perform variable size semi-major axis orbit increment maneuvers in order to keep the satellite ground track within its allowed limits $( \pm 4 \mathrm{~km})$.
\end{abstract}

Keywords: autonomous orbit control, GPS, autonomous navigator, Kalman Filter.

\section{Introduction}

In a former study [3] the performance of an autonomous orbit control procedure was analyzed considering the direct use, in the feedback loop, of the coarse GPS navigation solution. That work considered a hypothetical satellite, equipped with a GPS navigator receiver, placed in a phased helio-synchronous orbit. Under worst case conditions in terms of solar activity, considered in that investigation, the autonomous control successfully maintained the Equator longitude phase drift (D) restricted to an excursion range of about $-1000 \mathrm{~m}$ and $1700 \mathrm{~m}$. In a posterior study [1], an autonomous orbit control procedure applied to a CBERS-like simulated satellite was proposed and had its performance assessed. In that work, a simplified GPS navigator was used in the feedback control loop, in order to supply the needed autonomous orbit observations. The results of a longterm computer simulation (one year) indicated the feasibility of the application of the simplified GPS navigator to the autonomous orbit control system. Essentially, the simplified navigator consists of a Kalman filtering process which incorporates a procedure for automatic treatment of observation biases. The idea behind using a simplified navigator is to allow the computation of improved orbit estimates from the GPS (geometric) navigation solution, without adding a significant computational burden to the autonomous orbit control procedure. The introduction of the simplified navigator to the autonomous control procedure successfully improved the control results, significantly reducing the variation range of $\mathrm{D}$. Both realistic and worst case conditions in terms of solar activity were considered in the simulation.

The second study [1] was done considering a version of the autonomous orbit control procedure which considered only the application of semi-major axis corrections with a constant, previously chosen amplitude, while the first one [3] considered variable amplitude semi-major axis corrections. It is also worth mentioning that in [3] the raw observations of both $\mathrm{D}$ and its first time derivative, 
$\dot{\mathrm{D}}$, were computed from each simulated set of GPS orbit estimates, whereas in [1] only the D observations were computed from the orbit estimates while the $\dot{D}$ and $\ddot{D}$ observations were directly computed, in a numerical way, from the last computed observations of D. This last approach increased the accuracy of the $\dot{D}$ observations and, as a consequence, the performance of the autonomous control process.

It is well known that the acceleration of the orbit ground track drift $\ddot{D}$ depends on the solar activity conditions. In [2], a novel approach for predicting the effects of solar activity on the evolution of ground track drift of phased satellites was presented. It requires independent runs of a very realistic orbit simulator for the satellite considered, for several values of the geomagnetic activity index, $\mathrm{K}_{\mathrm{p}}$, and for several values of the solar flux, $\mathrm{F}_{10.7}$. It was noticed that a third to forth degree polynomials almost perfectly adjusted the drift acceleration $\ddot{D}$ as a function of $F_{10.7}$ data, for all values of $K_{p}$. As a result of the procedure given in [2], a set of polynomials in function of $\mathrm{K}_{\mathrm{p}}$ and $\mathrm{F}_{10.7}$ is obtained that make it possible to calculate $\ddot{D}$ for the satellite as long as values for $K_{p}$ and $F_{10.7}$ are provided.

In the present study, it is analyzed a version of the autonomous orbit control procedure that makes use of improved orbit estimates as provided by the simplified navigator proposed in [1] and makes use of variable amplitude semi-major axis corrections during the maneuvers, similar to what was done in [2]. Moreover, the approach presented in this paper uses the polynomial method proposed in [2] in order to calculate $\ddot{D}$. For that aim, it is considered that the onboard autonomous control system of the satellite allows receiving the required values of $\mathrm{K}_{\mathrm{p}}$ and $\mathrm{F}_{10.7}$ as inputs provided by internal sensors or by telecommand from soil. As a consequence of such approach, it is eliminated one of the greatest sources of uncertainty, namely, the value of $\ddot{D}$. The new approach also calculates the semi-major axis maneuver amplitude in order to maximize the time between consecutive maneuvers and minimizing, this way, the maneuver application number.

\section{Autonomous Control Procedure}

A block diagram of the autonomous control system considered in [1] and also in the present work is given in Figure 1. It gives an overview of the full simulation loop.

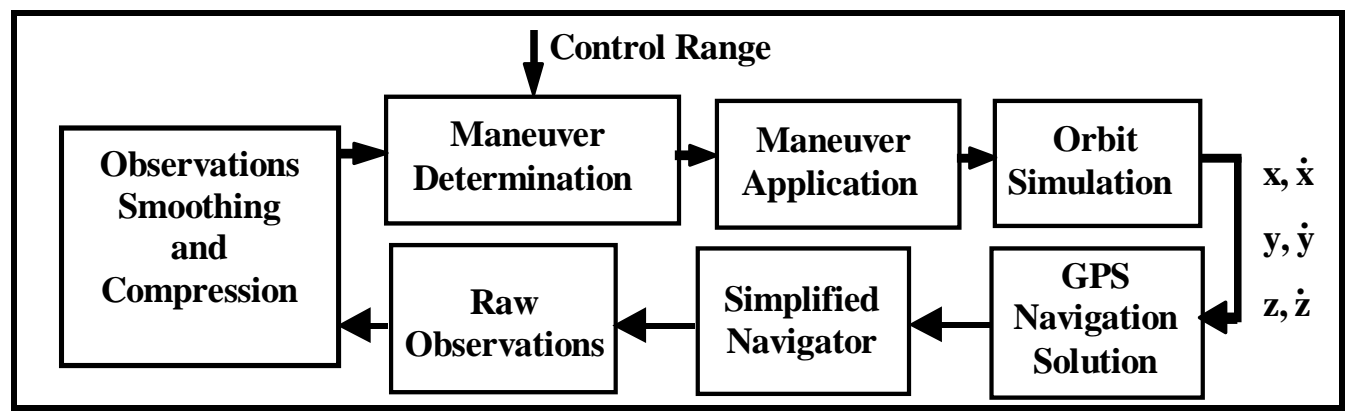

Figure 1. Block Diagram of the Autonomous Control System

Starting the diagram description in the block GPS Navigation Solution, the respective GPS estimates are computed with help of a realistic orbit simulation process. Typical root mean square errors of the coarse GPS geometric estimates were of $100 \mathrm{~m}$ in position and $1 \mathrm{~m} / \mathrm{s}$ in velocity, before Selective Availability was turned off. Added to such random errors these estimates presented systematic variations with values of the order of $100 \mathrm{~m}$ and duration of about 1 to 15 minutes. Both the random and systematic errors are considered in the simulation of GPS coarse navigation solution estimates used in this work and in the levels mentioned, since the idea is to compare its performance with previous studies. The GPS Simplified Navigator takes the position components of these estimates as inputs. Next, Raw Observations of D are computed from each set of improved orbit 
estimates supplied by the simplified navigator. These raw observations are preprocessed in real time in the block Observations Smoothing and Compression, in order to achieve data smoothing by curve fitting, validation and redundancy reduction. Also in this block, observations of $\dot{\mathrm{D}}$ are numerically calculated from the smoothed values of D. Finally, the computed observations of D and $\dot{D}$ are used within the Maneuver Determination process, where the instants of orbit correction applications are defined and their respective amplitudes calculated. In the case of the present control approach, information about $\mathrm{K}_{\mathrm{p}}$ and $\mathrm{F}_{10.7}$ are furnished to this block together with the control ranges. Once defined the need of a maneuver, its execution occurs within the block Maneuver Application, where its amplitude and the corresponding changes in the orbital parameters of the satellite are calculated and imposed. Closing the simulation loop, the block Orbit Simulation performs realistic orbit propagation, incorporating the orbit maneuvers to the propagation whenever the Maneuver Determination process determines its execution.

The first task of the autonomous orbit control process is the computation of raw observations of D from the orbit estimates issued by the simplified navigator. The following equation is used:

$$
\mathrm{D}=\mathrm{a}_{\mathrm{e}} \cdot\left[\Delta \Omega+\frac{\Delta \alpha}{(\mathrm{N}+\mathrm{P} / \mathrm{Q})}\right]
$$

where $\mathrm{a}_{\mathrm{e}}$ is the mean Equator radius, $\Delta \Omega$ is the right ascension of the ascending node deviation from the reference value; $\Delta \alpha$ is argument of latitude deviation from the reference value; $\mathrm{N}$ is the integer number of orbit revolutions per day; $\mathrm{P}$ and $\mathrm{Q}$ are two integers whose ratio determines the additional fraction of orbit completed by the satellite in one day. The argument of latitude itself is given by $\alpha=\omega+M$, the sum of the perigee argument and the mean anomaly of the satellite, respectively.

Next, the raw values of D are preprocessed in real time in the block Observations Smoothing and Compression, using a version of a weighted moving average procedure developed in [4], with the aim of filtering cyclic perturbations and data redundancy reduction. Mathematically:

For $\mathrm{k}<\mathrm{N}: \quad \overline{\mathrm{D}}\left(\mathrm{t}_{\mathrm{k}}\right)=\frac{\mathrm{S}_{1}+\mathrm{w}\left(\mathrm{t}_{\mathrm{k}}\right) \mathrm{D}\left(\mathrm{t}_{\mathrm{k}}\right)}{\mathrm{S}_{2}+\mathrm{w}\left(\mathrm{t}_{\mathrm{k}}\right)} ; \quad \mathrm{S}_{1}=\sum_{\mathrm{j}=0}^{\mathrm{k}-1} \mathrm{w}\left(\mathrm{t}_{\mathrm{j}}\right) \mathrm{D}\left(\mathrm{t}_{\mathrm{j}}\right) ; \quad \mathrm{S}_{2}=\sum_{\mathrm{j}=0}^{\mathrm{k}-1} \mathrm{w}\left(\mathrm{t}_{\mathrm{j}}\right)$

For $k>N: \bar{D}\left(t_{k}\right)=\frac{[(N-1) / N] \cdot S_{3}+w\left(t_{k}\right) D\left(t_{k}\right)}{[(N-1) / N] \cdot S_{4}+w\left(t_{k}\right)} ; S_{3}=\sum_{j=k-N}^{k-1} w\left(t_{j}\right) D\left(t_{j}\right) ; S_{4}=\sum_{j=k-N}^{k-1} w\left(t_{j}\right)$

where $\bar{D}\left(t_{k}\right)$ is the smoothed value for $D$ at instant $t_{k}$; $\left(t_{k}\right)$ is the raw value of $D$ at $t_{k}$; $w\left(t_{k}\right)$ is the weight factor of $D\left(t_{k}\right)$, and $N$ is a previously defined constant that represents the maximum number of observations to take part in the smoothing process. Equation 2 is used in the beginning of the process, when there are less than $\mathrm{N}$ observations and Equation 3 is used from there on. It is important to notice that, from one instant $\left(t_{k-1}\right)$ to another $\left(t_{k}\right)$, only the values of $S_{1}$ and $S_{2}$ or $S_{3}$ and $\mathrm{S}_{4}$ need to be stored. The weight factor can be taken as $\mathrm{w}\left(\mathrm{t}_{\mathrm{k}}\right)=1 /\left(\sigma\left(\mathrm{t}_{\mathrm{k}}\right)^{2}\right)$, where $\sigma\left(\mathrm{t}_{\mathrm{k}}\right)$ is the uncertainty associated to the observation $\mathrm{D}\left(\mathrm{t}_{\mathrm{k}}\right)$. In this work, the value of $\mathrm{N}$ is always calculated by $\mathrm{N}=\operatorname{INT}\left(\Delta \mathrm{t}_{\mathrm{SMT}} / \Delta \mathrm{t}_{\mathrm{C}}\right)$, where $\mathrm{INT}\left(\right.$.) means rounding to the nearest integer, $\Delta \mathrm{t}_{\mathrm{S}}$ is the "time window" of the observations considered in the smoothing process and $\Delta \mathrm{t}_{\mathrm{C}}$ is the time interval between two consecutive observations of $\mathrm{D}$. Considering $\Delta \mathrm{t}_{\mathrm{S}}$ as a parameter instead of $\mathrm{N}$ has the advantage of turning the smoothing process less dependent from $\Delta \mathrm{t}_{\mathrm{C}}$, since the same $\mathrm{N}$ implies different time windows for different $\Delta \mathrm{t}_{\mathrm{C}}$. Considering that most perturbations one wishes to filter by the smoothing process are cyclic ones, using $\Delta \mathrm{t}_{\mathrm{S}}$ avoid having to recalculate $\mathrm{N}$ manually when employing different $\Delta \mathrm{t}_{\mathrm{C}}$ 's and facilitating, this way, the parameter setting for the autonomous 
control system. Whenever one orbit correction is applied to the satellite, the smoothing procedure just described is reinitiated by imposing $S_{1}=S_{2}=S_{3}=S_{4}=0$.

Values of $\dot{\mathrm{D}}$ are also numerically calculated in the same block, using:

$$
\dot{\mathrm{D}}\left(\mathrm{t}_{\mathrm{k}}\right)=\left(\overline{\mathrm{D}}\left(\mathrm{t}_{\mathrm{k}}\right)-\overline{\mathrm{D}}\left(\mathrm{t}_{\mathrm{k}-1}\right)\right) /\left(\mathrm{t}_{\mathrm{k}}-\mathrm{t}_{\mathrm{k}-1}\right)
$$

These values are smoothed further by using the same procedure just described for D (Eq. 2 and 3), generating $\dot{\overline{\mathrm{D}}}\left(\mathrm{t}_{\mathrm{k}}\right)$. If one assumes constant solar flux during the time interval between the application of two successive orbit correction maneuvers, which implies in having constant a (a being the orbit semi-major axis), the time evolution curve of $\mathrm{D}$ is almost parabolic, and Equation 5 next can be used by the maneuver computation process, to foresee the time evolution of the Equator longitude phase drift.

$$
\overline{\mathrm{D}}(\mathrm{t})=\overline{\mathrm{D}}\left(\mathrm{t}_{\mathrm{k}}\right)+\dot{\overline{\mathrm{D}}}\left(\mathrm{t}_{\mathrm{k}}\right) \Delta \mathrm{t}+1 / 2 \ddot{\overline{\mathrm{D}}}\left(\mathrm{t}_{\mathrm{k}}\right)(\Delta \mathrm{t})^{2}
$$

where $\Delta t=t-t_{k}$ is the elapsed time since $t_{k}$. The value of $\dot{\bar{D}}\left(t_{k}\right)$ is known from Eq. 4 and the value of $\ddot{\overline{\mathrm{D}}}\left(\mathrm{t}_{\mathrm{k}}\right)$ is calculated using the polynomial method proposed in [2]. The estimates $\overline{\mathrm{D}}\left(\mathrm{t}_{\mathrm{k}}\right), \dot{\overline{\mathrm{D}}}\left(\mathrm{t}_{\mathrm{k}}\right)$ and $\ddot{\bar{D}}\left(t_{k}\right)$ are used by the block Maneuver Determination to determine the need of maneuvers and to compute the required correction amplitudes. One maneuver is considered needed when any of the following two conditions is verified:

$$
\begin{gathered}
\overline{\mathrm{D}}\left(\mathrm{t}_{\mathrm{k}}\right)>\mathrm{D}_{\max }-\mathrm{n} . \sigma\left(\mathrm{t}_{\mathrm{k}}\right) \\
\overline{\mathrm{D}}\left(\mathrm{t}_{\mathrm{k}+1}\right)>\mathrm{D}_{\max }-\mathrm{n} . \sigma\left(\mathrm{t}_{\mathrm{k}}\right)
\end{gathered}
$$

where $D_{\max }$ is a previously chosen control limit; $\sigma\left(t_{k}\right)$ is the standard deviation of $\bar{D}\left(t_{k}\right) ; t_{k}$ is the time instant of the last (k-th) observation sample known and $\mathrm{n}$ is a real number. The future estimate for $\bar{D}\left(t_{k+1}\right)$ is calculated using Eq. 5. The idea behind Eq. 7 is to anticipate a maneuver if it is foreseen that in the next maneuver verification moment, i.e., $t_{k+1}$, the value estimated for the ground track drift is greater than the allowed limit. If that happens, Eq. 7 allows the maneuver to occur at instant $t_{k}$.

Only the application of positive corrections to the orbit semi-major axis is considered for the maintenance of D inside the control ranges. Each semi-major axis increment orbit is computed in order to change the value of $\dot{D}$ such that the minimal value to occur for $D$ after the maneuver equals a previously chosen inferior limit, $\mathrm{D}_{\min }$. The maximization of the time interval between the executions of two successive maneuvers is implicit in this strategy.

Assuming that $\mathrm{D}_{\min }$ occurs at $\mathrm{t}=\mathrm{t}_{\min }$ after a maneuver occurred at $\mathrm{t}=\mathrm{t}_{\mathrm{M}}$ and taking this to Eq. 5 at $\mathrm{t}=\mathrm{t}_{\mathrm{M}}^{+}$, a time instant right after the maneuver, one arrives at:

$$
1 / 2 \ddot{\overline{\mathrm{D}}}\left(\mathrm{t}_{\mathrm{M}}^{+}\right)(\Delta \mathrm{t})^{2}+\dot{\overline{\mathrm{D}}}\left(\mathrm{t}_{\mathrm{M}}^{+}\right) \Delta \mathrm{t}+\left(\overline{\mathrm{D}}\left(\mathrm{t}_{\mathrm{M}}^{+}\right)-\mathrm{D}_{\text {min }}\right)=0
$$

where $\Delta \mathrm{t}=\mathrm{t}_{\mathrm{min}^{-}}-\mathrm{t}_{\mathrm{M}}^{+}$is the unknown. The solution of Eq. 8 for $\Delta \mathrm{t}$ is: 


$$
\Delta \mathrm{t}=\left(-\dot{\overline{\mathrm{D}}}\left(\mathrm{t}_{\mathrm{M}}^{+}\right) \pm \sqrt{\left(\dot{\overline{\mathrm{D}}}\left(\mathrm{t}_{\mathrm{M}}^{+}\right)\right)^{2}-2 \ddot{\overline{\mathrm{D}}}\left(\mathrm{t}_{\mathrm{M}}^{+}\right)\left(\overline{\mathrm{D}}\left(\mathrm{t}_{\mathrm{M}}^{+}\right)-\mathrm{D}_{\text {min }}\right)}\right) /\left(\ddot{\overline{\mathrm{D}}}\left(\mathrm{t}_{\mathrm{M}}^{+}\right)\right)
$$

Realizing that $\mathrm{D}=\mathrm{D}_{\min }$ must occur just once, it means that there is only one root for Eq. 9 what implies that the square root in Eq. 9 must be equal to zero. Mathematically:

$$
\begin{gathered}
\sqrt{\left(\dot{\overline{\mathrm{D}}}\left(\mathrm{t}_{\mathrm{M}}^{+}\right)\right)^{2}-2 \ddot{\overline{\mathrm{D}}}\left(\mathrm{t}_{\mathrm{M}}^{+}\right)\left(\overline{\mathrm{D}}\left(\mathrm{t}_{\mathrm{M}}^{+}\right)-\mathrm{D}_{\min }\right)}=0 \\
\Delta \mathrm{t}=-\dot{\overline{\mathrm{D}}}\left(\mathrm{t}_{\mathrm{M}}^{+}\right) /\left(\ddot{\overline{\mathrm{D}}}\left(\mathrm{t}_{\mathrm{M}}^{+}\right)\right)=\Rightarrow \mathrm{t}_{\text {min }}=\mathrm{t}_{\mathrm{M}}-\dot{\overline{\mathrm{D}}}\left(\mathrm{t}_{\mathrm{M}}^{+}\right) /\left(\ddot{\overline{\mathrm{D}}}\left(\mathrm{t}_{\mathrm{M}}^{+}\right)\right)
\end{gathered}
$$

In Eq. 8, the values of $\overline{\mathrm{D}}\left(\mathrm{t}_{M}^{+}\right)$and $\ddot{\overline{\mathrm{D}}}\left(\mathrm{t}_{M}^{+}\right)$can be taken for $\mathrm{t}=\mathrm{t}_{\mathrm{M}}$ instead of $\mathrm{t}=\mathrm{t}_{\mathrm{M}}^{+}$, what means they are known. For $\dot{\overline{\mathrm{D}}}\left(\mathrm{t}_{\mathrm{M}}^{+}\right)$Eq. 10 can be rearranged such that:

$$
\dot{\overline{\mathrm{D}}}\left(\mathrm{t}_{\mathrm{M}}^{+}\right)=-\sqrt{2 \ddot{\overline{\mathrm{D}}}\left(\mathrm{t}_{\mathrm{M}}^{+}\right)\left(\overline{\mathrm{D}}\left(\mathrm{t}_{\mathrm{M}}^{+}\right)-\mathrm{D}_{\text {min }}\right)}=-\sqrt{2 \ddot{\overline{\mathrm{D}}}\left(\mathrm{t}_{\mathrm{M}}\right)\left(\overline{\mathrm{D}}\left(\mathrm{t}_{\mathrm{M}}\right)-\mathrm{D}_{\text {min }}\right)}
$$

The minus sign in Eq. 12 instead of \pm comes from the fact that, after the maneuver, one desires $\dot{D}<$ 0 in order to revert the natural increasing tendency of D (natural Eastward longitude ground track drift). Considering some approximations which can be assumed for phased helio-synchronous orbits like those of CBERS satellites, the maneuver size in terms of semi-major axis variation, $\Delta \mathrm{a}$, is calculated by [5]:

$$
\Delta \mathrm{a}=-\frac{\mathrm{T}_{\mathrm{te}}}{3 \pi} \frac{\mathrm{a}_{\mathrm{R}}}{\mathrm{a}_{\mathrm{e}}}\left(\dot{\overline{\mathrm{D}}}\left(\mathrm{t}_{\mathrm{M}}^{+}\right)-\dot{\overline{\mathrm{D}}}_{\mathrm{C}}\left(\mathrm{t}_{\mathrm{M}}^{-}\right)\right)
$$

where $\dot{\overline{\mathrm{D}}}\left(\mathrm{t}_{\mathrm{M}}^{+}\right)$is calculated by Eq. $12 ; \dot{\overline{\mathrm{D}}}_{\mathrm{C}}\left(\mathrm{t}_{\mathrm{M}}^{-}\right)$is the last preprocessed value of $\dot{\overline{\mathrm{D}}}\left(\mathrm{at} \mathrm{t}=\mathrm{t}_{\mathrm{M}}\right.$ ); $\mathrm{a}_{\mathrm{R}}$ is the semi-major axis of the reference orbit and $a_{e}$ is the mean Equator radius. The corresponding tangential velocity increment, $\Delta \mathrm{V}_{\mathrm{T}}$, is calculated by:

$$
\Delta \mathrm{V}_{\mathrm{T}}=\frac{\Delta \mathrm{a}}{2 \mathrm{a}_{\mathrm{R}}} \mathrm{V}
$$

where $\mathrm{V}$ is the magnitude of the velocity vector of the satellite.

\section{Autonomous Control Test Results}

The performance of the autonomous orbit control procedure just proposed was verified through the execution of a realistic simulation of its application to a CBERS-like satellite. In the same way it was done in [1], the simulation covered a period of about one-year and considering two scenarios in terms of solar activity. Realistic (moderate) and critical solar activity conditions were considered in the analysis. In the moderate solar flux profile, a maximum of $165 \mathrm{sfu}$, in the beginning, and a minimum of $115 \mathrm{sfu}$, in the end, with 27-day cycle oscillations of about $25 \mathrm{sfu}$ was used for the solar activity simulation in [1] and here. In the critical solar flux profile, the 11-year cycle is compressed to one-year, with a very high maximum of about 300 solar flux units (sfu) and the 27day cycle oscillations due to solar rotation with amplitudes of $80 \mathrm{sfu}$. In [1], a maximum of $360 \mathrm{sfu}$ was used. Due to the fact that the polynomial approach presented in [2] has 300 as the maximum possible value for the solar flux, in the present study, one changed the critical solar flux profile to match this restriction. This was done lowering the corresponding profile used in [1] by a value of 60 
sfu. A maximal application rate of about one maneuver per orbit period ( 100 min) was considered. It was also considered a GPS observation rate (and consequently the navigator output rate) of 1 estimate each 9 seconds. Only one among 20 orbit estimates sets successively issued by the navigator is used by the control system (meaning a rate of one data each three minutes, e.g., $\Delta \mathrm{t}_{\mathrm{C}}=$ $3 \mathrm{~min})$. For all cases tested, the weight factors $w\left(\mathrm{t}_{\mathrm{k}}\right)$ used by Eq.s 2 and 3 were all set to 1 . After each maneuver the smoothing of $\mathrm{D}$ and $\dot{\mathrm{D}}$ was restarted (by imposing $\mathrm{k}=0$ in Eq.s 2 and 3) and a time interval without maneuvers of $24 \mathrm{~h}$ was observed. This last measure was implemented in order to allow the effects on D from a maneuver just applied to become measurable and avoiding, this way, the premature application of a second maneuver.

The results of the current work, considering moderate solar activity condition, are shown in Fig.s 2 and 3 for the ground track drift time evolution and the semi-major axis maneuvers, respectively. The same observation rate $\left(\Delta \mathrm{t}_{\mathrm{C}}=3 \mathrm{~min}\right)$ and the same maximal allowable maneuver application rate used in [1] were considered. The values $D_{\max }=3,900 \mathrm{~m}$ and $\mathrm{n}=0$ were used in Eq.s 6 and 7, and $\mathrm{D}_{\min }=-$ $3,900 \mathrm{~m}$ in Eq. 12. For $\Delta \mathrm{t}_{\mathrm{s}}$, the values $3 \mathrm{~h}$ and $6 \mathrm{~h}$ were used for smoothing $\mathrm{D}$ and $\dot{\mathrm{D}}$, respectively. The number of maneuvers was 17 with an accumulated $\Delta$ a for the period of $2,092.4 \mathrm{~m}$. The major maneuver had $\Delta \mathrm{a}=175.9 \mathrm{~m}$ and the minor $\Delta \mathrm{a}=45.0 \mathrm{~m}$. From Fig. 2, it is possible to see that, for the moderate solar activity profile, the new autonomous control version was able to make full use of the $\pm 4 \mathrm{~km}$ allowed range for $\mathrm{D}$ in order to reduce the amount of applied maneuvers.

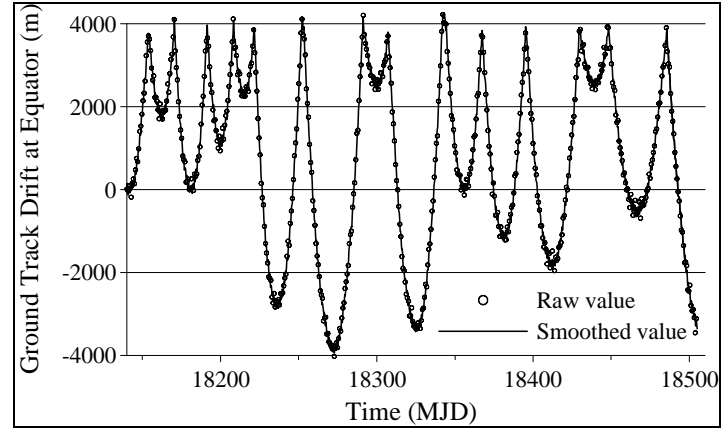

Figure 2. Ground Track Drift

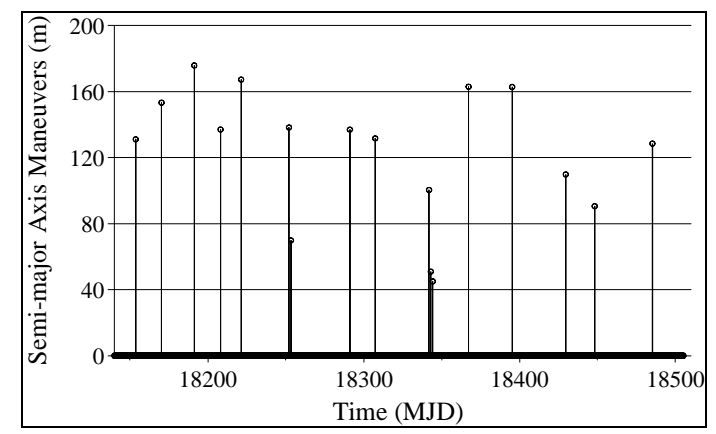

Figure 3. Semi-major Axis Maneuvers

In order to assess the performance of the new control approach proposed here in terms of its ability to keep $D$ under very stringent requirements, the values $D_{\max }=100 \mathrm{~m}$ and $D_{\min }=-100 \mathrm{~m}$ were used to generate the results shown in Fig. 4. For $\Delta \mathrm{t}_{\mathrm{S}}$, the values used were $8 \mathrm{~h}$ and $20 \mathrm{~h}$ for smoothing $\mathrm{D}$ and $\dot{\mathrm{D}}$, respectively. The number of maneuvers was 146 with an accumulated $\Delta$ a for the whole period of $2,178.7 \mathrm{~m}$. The major maneuver had $\Delta \mathrm{a}=23.9 \mathrm{~m}$ and the minor $\Delta \mathrm{a}=6.1 \mathrm{~m}$. As for comparison, the result obtained in [1] for the same solar activity and maximal allowable maneuver rate is given in Fig. 5. There, the autonomous orbit control procedure considered only the application of semi-major axis corrections with constant amplitude equal to $3 \mathrm{~m}$. The number of applied maneuvers was 666 with $\Delta \mathrm{a}=3 \mathrm{~m}$ each, resulting an accumulated total of $1,998 \mathrm{~m}$ for the whole period. As can be seen by comparing Fig.s 4 and 5, both control versions have similar performances in terms of keeping D within a tight range, with a slight advantage for the version presented in this paper. In terms of accumulated $\Delta \mathrm{a}$, the control version from [1] was about $9 \%$ smaller. Finally, in terms of the number of applied maneuvers, the control version presented now applied about $22 \%$ of the number of maneuvers applied in [1]. 


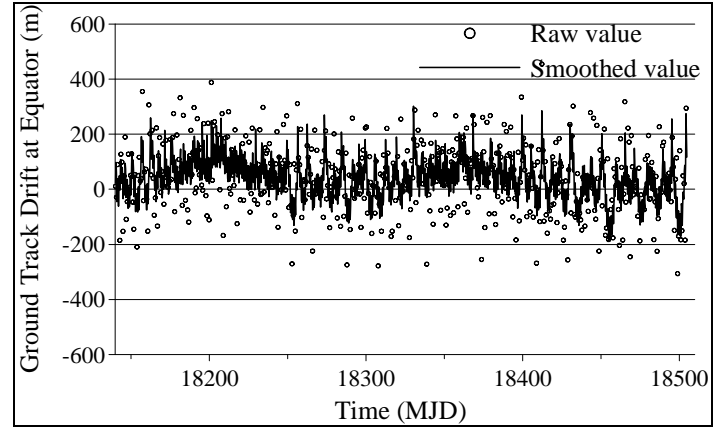

Figure 4. Ground Track Drift

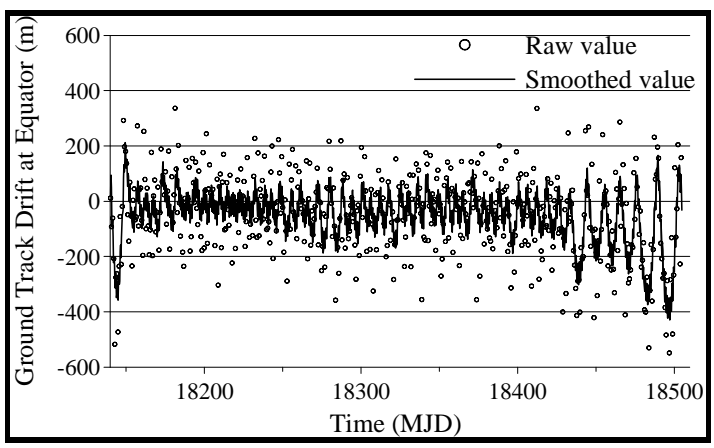

Figure 5. Ground Track Drift from [1]

The results of the current work, considering critical solar activity condition, are shown in Fig.s 6 and 7 for the ground track drift time evolution and the semi-major axis maneuvers, respectively. The same observation rate $\left(\Delta \mathrm{t}_{\mathrm{C}}=3 \mathrm{~min}\right)$ and the same maximal allowable maneuver application rate used in [1] were considered. The values $D_{\max }=3,900 m$ and $n=0$ were used in Eq.s 6 and 7, and $D_{\min }=-$ 3,900m in Eq. 12. For $\Delta \mathrm{t}_{\mathrm{S}}$, the values $8 \mathrm{~h}$ and $40 \mathrm{~h}$ were used for smoothing $\mathrm{D}$ and $\dot{\mathrm{D}}$, respectively. The number of maneuvers was 26 with an accumulated $\Delta \mathrm{a}$ for the period equal to $2,953.0 \mathrm{~m}$. The major maneuver had $\Delta \mathrm{a}=324.5 \mathrm{~m}$ and the minor $\Delta \mathrm{a}=21.7 \mathrm{~m}$. From Fig. 6, it is possible to see that, for the critical solar activity profile, the new autonomous control version was able to make almost full use of the $\pm 4 \mathrm{~km}$ allowed range for $\mathrm{D}$ in order to reduce the amount of applied maneuvers.

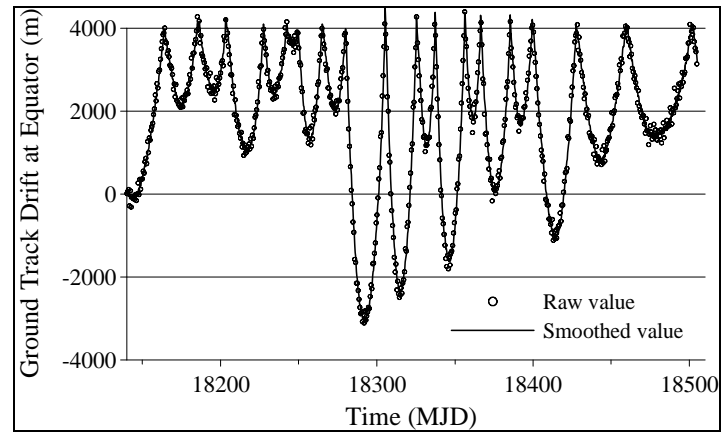

Figure 6. Ground Track Drift

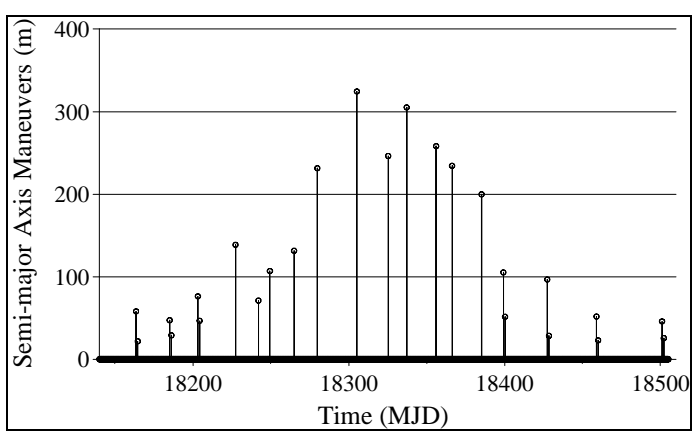

Figure 7. Semi-major Axis Maneuvers

The results obtained when the values $D_{\max }=100 \mathrm{~m}$ and $\mathrm{D}_{\min }=-100 \mathrm{~m}$ were used are given in Fig. 8 . For $\Delta \mathrm{t}_{\mathrm{s}}$, the values used were $8 \mathrm{~h}$ and $25 \mathrm{~h}$ for smoothing $\mathrm{D}$ and $\dot{\mathrm{D}}$, respectively. The number of maneuvers was 148 with an accumulated $\Delta$ a for the whole period of $2,946.7 \mathrm{~m}$. The major maneuver had $\Delta \mathrm{a}=64.0 \mathrm{~m}$ and the minor $\Delta \mathrm{a}=3.6 \mathrm{~m}$. As for reference, the result obtained in [1] for similar solar activity and the same maximal allowable maneuver rate is given in Fig. 9. There, semi-major axis corrections with constant amplitude equal to $4 \mathrm{~m}$ were used. The number of applied maneuvers was 1,145 with $\Delta \mathrm{a}=4 \mathrm{~m}$ each, resulting an accumulated total of $4,580 \mathrm{~m}$ for the whole period. Unfortunately, a direct comparison among the results obtained here with those from [1] is not possible, since they have used different levels of critical solar profiles. 


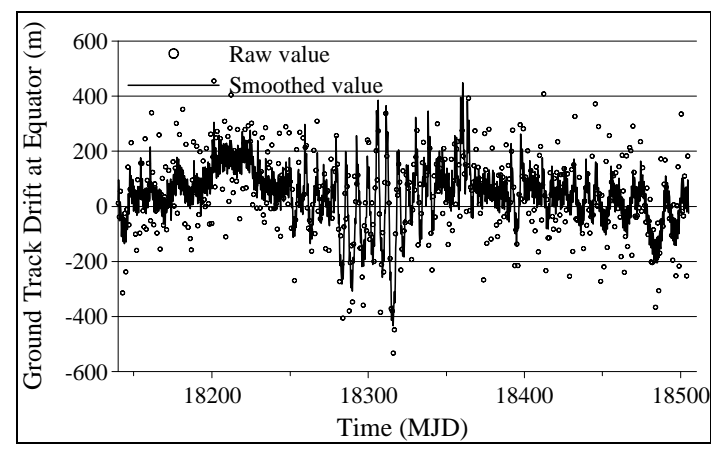

Figure 8. Ground Track Drift

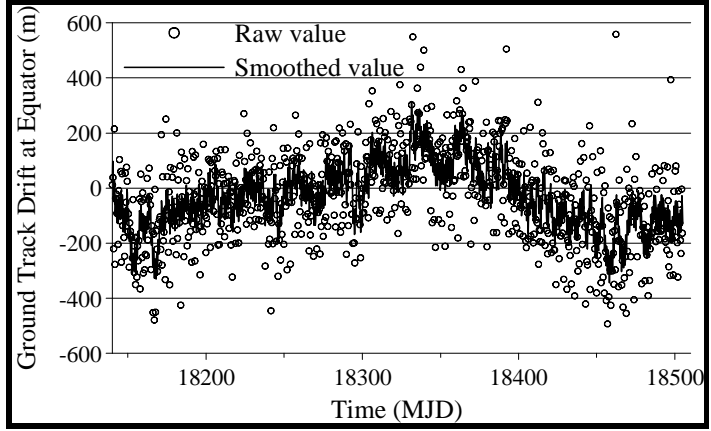

Figure 9. Ground Track Drift from [1]

\section{Conclusions}

In this article, it was analyzed a version of an autonomous orbit control procedure that makes use of improved orbit estimates provided by a simplified navigator and uses variable amplitude semi-major axis corrections in order to keep the ground track drift at equator of a CBERS-like sun-synchronous satellite within its allowed variation range. A polynomial approach recently proposed to calculate the second time derivative of the ground track drift was also used. The main conclusion is that the objectives were successfully achieved. The new method used to calculate the second derivative of the ground track drift helped reducing the uncertainty present in this parameter, allowing more precise calculations of the semi-major axis maneuver amplitudes and contributing to reduce the number of applied maneuvers, as originally desired. The obtained results can be considered very positive. They revealed that the new approach is advantageous, in terms of the number of maneuvers applied, even when tight operational limits are considered. In this way, the main objective of presenting a method capable of minimizing the number of maneuvers by full use of the allowed range for the ground track drift was completely fulfilled.

\section{References}

[1] Galski, R. L.; Orlando, V.; and Kuga, H. K., "Autonomous Orbit Control Procedure, Using a Simplified GPS Navigator, Applied to the CBERS Satellite", In: 16th International Symposium on Spaceflight Dynamics, 2001, Pasadena. CD-ROM of 16th ISSFD, 2001.

[2] Orlando, V.; Kuga, H. K.; and Galski, R. L., “An Approach for Predicting the Effects of Solar Activity on the Evolution of Ground Track Drift of Phased Satellites", In: 21st International Symposium on Space Flight Dynamic - 21st ISSFD, 2009, Toulouse - France, 21st International Symposium on Space Flight Dynamic, Toulouse - France : CNES, pp. 1-12, 2009.

[3] Orlando, V. and Kuga, H. K., "Analysis of an Autonomous Orbit Control Concept Using GPS", Journal of the Brazilian Society of Mechanical Sciences, Vol. XXI, Special Issue, pp. 52-59, February, 1999.

[4] Galski, R. L., "Autonomous Orbit Navigator Development, Using Gps, Applied to Autonomous Orbit Control”, 162 p. (INPE-8982-TDI/813). (In Portuguese). Master Degree Dissertation on Space Mechanics and Control - Instituto Nacional de Pesquisas Espaciais, São José dos Campos, Brazil. 2001. Available at: <http://urlib.net/sid.inpe.br/jeferson/2004/09.01.11.28>.

[5] Micheau, P., "Orbit Control Techniques for Low Earth Orbiting Satellites", Space Flight Dynamics (chap. 13), CNES, France, CEPADUES Editions, 1995. 